\title{
QUEBRA DE DORMÊNCIA DE PEREIRA ‘HOSUI’ COM USO DE ÓLEO MINERAL EM DOIS TIPOS DE CONDUÇÃO'
}

\author{
ODIRLEI RAIMUNDO DE OLIVEIRA ${ }^{2}$, RAFAELAPARECIDO PERESSUTI $^{2}$, REBERT SKALITZ $^{2}$, \\ MARINA COSTACURTA ANTUNES ${ }^{2}$, LUIZ ANTONIO BIASI ${ }^{3}$, FLÁVIO ZANETTE ${ }^{3}$
}

RESUMO - Algumas cultivares de pereira asiática, quando plantadas em regiões onde ocorrem invernos com baixo número de horas de frio, comumente apresentam deficiência e desuniformidade na brotação e floração, necessitando de meios artificiais para a quebra de dormência. Visando a miminizar esse problema, foi instalado um experimento num pomar de 4 anos de pereiras 'Hosui' (Pyrus pyrifolia), em espaçamento 4,0m x 4,0m, na Estação Experimental do Canguiri-da Universidade Federal do Paraná, no município de Pinhais-PR (latitude $25^{\circ} 25^{\prime}$ sul, longitude $49^{\circ} 08^{\prime}$ e altitude $920 \mathrm{~m}$ ), onde a soma de horas de frio atingiu 141 horas abaixo de $7,2^{\circ} \mathrm{C}$ durante o inverno de 2005. Os tratamentos foram aplicados quando as plantas estavam no estádio fenológico $\mathrm{A}_{1}$ (gema inchada), sendo o experimento realizado em delineamento inteiramente ao acaso, com 4 repetições, em esquema fatorial $2 \times 4 \times 7$, onde foram testados dois tipos de condução, em taça e em líder central modificado, três concentrações de óleo mineral a 4\%, 6\% e 8\%, e uma testemunha, durante o período de agosto a novembro de 2005 , e sete avaliações realizadas semanalmente pelo número de gemas em início de brotação, gemas brotadas e gemas mortas, em 4 ramos de um ano de idade e em posição inclinada, previamente marcados por planta. Os resultados obtidos mostraram que a aplicação de óleo mineral, nas concentrações de $4 \%, 6 \%$ e $8 \%$, anteciparam a brotação de gemas em relação à testemunha e aumentaram a porcentagem de gemas brotadas nos dois tipos de condução, sendo que as concentrações de $6 \%$ e $8 \%$ foram mais efetivas para antecipar a brotação do que a de $4 \%$. A porcentagem de gemas brotadas aos 84 dias após a aplicação do óleo mineral não diferiu entre os sistemas de condução, em líder central modificado e em taça.

Termos para indexação: Pyrus pyrifolia, horas de frio, indutores de brotação, produção orgânica, tipos de condução.

\section{DORMANCY BROKEN OF 'HOSUI' PEAR TREES WITH MINERAL OIL IN TWO TRAINING SYSTEMS}

\begin{abstract}
Some Asian pear cultivars, if cultivated in regions where less chilling hours are accumulated during winter, may present uneven budding and blooming and hence, need to have their dormancy broken by artificial means. In order to minimize such problem, an experiment was conducted in a 4-year-old 'Hosui' pear (Pyrus pyrifolia) with 4.0 x $4.0 \mathrm{~m}$ tree spacing orchard, at the Canguiri Station, in Pinhais-PR (latitude $25^{\circ} 25^{\prime}$ S, longitude $49^{\circ} 08^{\prime}$ and altitude of $920 \mathrm{~m}$ ), pertaining to the Federal University of Parana, in which accumulation of 141 chilling hours under $7.2^{\circ} \mathrm{C}$ was reached during the 2005 winter. Treatments were applied when plants were at the A1 phenological stage (bud swelling), using a completely randomized factorial design with 4 replications in a $2 \times 4 \times 7$ factorial scheme in which two types of tree training, vase or central leader, three mineral oil concentrations, 4,6 or $8 \%$, and a control applied from August to November 2005, and seven weekly performed evaluations based on the number of buds at pre-budding stage as well as broken and dead buds in four previously selected horizontally inclined 1-year-old stems per tree were tested. Obtained results showed that application of mineral oil at 4, 6 and $8 \%$ anticipated bud break compared to the control and enhanced bud break percentage in both training systems, being the concentrations of $6 \%$ and $8 \%$ more effective in anticipating bud break than $4 \%$. The percentage of bud break at the $84^{\text {th }}$ day after mineral oil application did not differ between the two training systems, i.e. vase and central leader.
\end{abstract}

Index terms: Pyrus pyrifolia, chilling hours, shoot inducers, organic production, training systems.

\section{INTRODUÇÃO}

A região metropolitana de Curitiba apresentou 161,8 horas de frio $\left(\leq 7,2^{\circ} \mathrm{C}\right)$, acumulada nos meses de maio a setembro, como valor médio dos anos de 2000 até 2004 (Botelho et al., 2006a). Nessa condição, as pereiras asiáticas (Pyrus pyrifolia) apresentam deficiência e desuniformidade na brotação e floração, pois o fornecimento de frio invernal encontra-se abaixo da exigência da maioria das cultivares, que está entre 300 a 900 horas, sendo que a cv. Housui necessita de 721 horas de frio para superar a dormência (Faoro, 2001). Em conseqüência disso, os ramos de crescimento do ano anterior apresentam muitas gemas que permanecem dormentes, mesmo que as condições sejam favoráveis ao crescimento, diminuindo o potencial de produção nos anos futuros (Petri et al., 1996).

Nas plantas cultivadas em regiões com inverno ameno, observa-se baixa taxa de brotação e florescimento heterogêneo,

1(Trabalho 107-07). Recebido em: 19-04-2007. Aceito para publicação em: 09-11-2007.

${ }^{2}$ Alunos do Curso de Agronomia da Universidade Federal do Paraná. Caixa Postal 19061. CEP 81531-990. Curitiba-PR. E-mail: odiufpr@pop.com.br, rperessuti@ibest.com.br,rtska@hotmail.com,marinacostacurta@hotmail.com.

${ }^{3}$ Professor do Departamento de Fitotecnia e Fitossanitarismo. Setor de Ciências Agrárias. Universidade Federal do Paraná. Caixa Postal 19061. CEP 81531-990. Curitiba-PR. Bolsista de Produtividade em Pesquisa do CNPq. E-mail: biasi@ufpr.br, flazan@ufpr.br. 
tanto no tempo como espacialmente ao longo do ramo (Leite, 2005). Além desses sintomas clássicos, ocorrem diversas anomalias, relacionadas com a falta de frio invernal, durante o ciclo vegetativo, nas gemas que conseguiram brotar, destacandose a paralisia no desenvolvimento do ramo, folhas pequenas, frutos pequenos e achatados e baixa frutificação efetiva (Petri \& Leite, 2004).

As fruteiras de clima temperado caracterizam-se pela queda das folhas no final do ciclo e a conseqüente entrada em dormência. Essa inatividade fisiológica permite a sobrevivência em condições de baixas temperaturas, proporcionando condições naturais para que iniciem um novo ciclo vegetativo na primavera (Leite, 2005). A inatividade das gemas é regulada por um balanço entre substâncias inibidoras de crescimento, como o ácido abscísico, e substâncias promotoras de crescimento, como citocininas e giberelinas (Taiz \& Zeiger, 1998). Durante esse período, a planta não demonstra crescimento visual, porém as atividades metabólicas continuam, embora com intensidade reduzida. Quando a soma de horas de frio não satisfaz a necessidade da cultura, faz-se necessária a quebra artificial da dormência, com a utilização de produtos químicos, viabilizando o cultivo de fruteiras de clima temperado em locais com baixo somatório de horas de frio invernal (Petri et al., 1996).

Vários compostos à base de nitrogênio já foram testados e mostraram-se efetivos na indução e brotação de pomáceas e frutas de caroço, porém apresentaram risco de fitotoxidez. O thidiazuron e o nitrato de cálcio, que foram testados para a quebra de dormência em macieira 'Gala', demonstraram boa eficiência quando aplicados juntamente com a cinaminada hidrogenada e óleo mineral (Nachtigal \& Camelatto, 1997). O thidiazuron 100 mg. $L^{-1}$ mais óleo mineral a $4 \%$ apresentou o mesmo efeito do que a cianamida hidrogenada a $0,5 \%$ mais óleo mineral a $4 \%$ na brotação de macieira 'Fuji' (Araújo et al., 1991). O thidiazuron também foi testado para a pereira e, em combinação com óleo mineral, promoveu a brotação de gemas laterais e terminais (Francisconi et al., 1992). Outros compostos, como querosene, calda sulfocálcica, nitrato de cálcio, Acordex e Erger, também foram testados como indutores de brotação em macieira 'Gala' e 'Fuji', sendo que Erger, mais nitrato de cálcio apresentaram-se mais eficientes e semelhantes ao uso do tratamento-padrão com óleo mineral mais cianamida hidrogenada. Já, o óleo mineral mais calda sulfocálcica ou querosene mostraram-se pouco eficientes na brotação de gemas axilares (Petri, 2005). No entanto, alguns desses indutores podem servir como alternativas para a quebra de dormência em cultivos orgânicos ou agroecológicos. A aplicação de extrato de alho (EA) mais óleo mineral (OM) em macieira 'Royal Gala' apresentou resultados similares ao tratamento com óleo mineral mais cianamida hidrogenada, sendo que o tratamento EA $10 \%+$ OM $2 \%$ atingiu $95 \%$ de gemas brotadas, além de antecipar a florada e a maturação dos frutos (Botelho et al., 2006b).

Devido à escassez de resultados de pesquisa local, com relação ao efeito do óleo mineral na quebra de dormência da pereira, este estudo foi realizado para testar sua eficiência na indução da brotação de gemas laterais, bem como a viabilização dessa prática para cultivos orgânicos.

\section{MATERIAL E MÉTODOS}

O experimento foi realizado num pomar de pereira asiática (Pyrus pyrifolia), cv. Hosui, de 4 anos de idade, com espaçamento de 4,0 $\mathrm{m}$ x 4,0 m, sendo utilizada como polinizadora a cv. Ya Li, na proporção de 4:1 e sobreenxerto de pereira cv. Kieffer em todas as plantas, localizado na Estação Experimental do Canguiri - da Universidade Federal do Paraná, no município de Pinhais-PR, situado a uma altitude de $920 \mathrm{~m}$, latitude $25^{\circ} 25^{\prime} \mathrm{S}$ e longitude $49^{\circ} 08^{\prime} \mathrm{W}$, durante o período de agosto a novembro de 2005 . O solo da área experimental é classificado como Latossolo VermelhoAmarelo, de textura argilosa.

O delineamento experimental utilizado foi o inteiramente casualizado, com quatro repetições, sendo analisado como em esquema fatorial $2 \times 4 \times 7$, onde foram testados dois tipos de condução, em taça e em líder central modificado, quatro concentrações de óleo mineral, a 4\%, 6\%,8\%, mais uma testemunha, e 7 datas de avaliação.

A unidade experimental foi constituída de uma planta, onde foram analisados quatro ramos de um ano em cada uma. Os ramos foram selecionados com, no mínimo, 12 gemas cada, sendo que nestes ramos foram contatos o número total de gemas laterais, gemas com início de brotação (ponta verde), gemas brotadas (com folhas visíveis) e gemas mortas.

A data de aplicação foi dia 22 de agosto de 2005, quando as gemas se encontravam no estádio fenológico $\mathrm{A}_{1}$ (gema inchada), e como fonte de óleo mineral usou-se a marca comercial Assist ${ }^{\circledR}$ (756 g.L L $^{-1}$ de ingrediente ativo). Um mês antes da aplicação do óleo mineral, as plantas foram podadas, pulverizadas com calda sulfocálcica a $10 \%$ e receberam adubação com esterco de carneiro.

As soluções de óleo mineral foram preparadas momentos antes de serem usadas, sendo aplicadas uma única vez em cada planta, com um pulverizador costal, molhando-se todos os ramos das plantas até o ponto de gotejamento e gastando-se, em média, 2 litros de solução por planta.

As avaliações ocorreram semanalmente, no período de 07 de outubro a 17 de novembro de 2005. Os dados obtidos foram submetidos à análise de variância e, quando verificada significância, procedeu-se à comparação de médias, pelo teste de Duncan, a 5\% de probabilidade, e pela análise de regressão polinomial.

\section{RESULTADOS E DISCUSSÃO}

Não houve interação entre os tipos de condução e as épocas de avaliação para as variáveis gemas em início de brotação e gemas brotadas, mas houve interação entre os tratamentos aplicados e as épocas de avaliação. Desta forma, foi realizada a análise de regressão para observar a tendência dessas variáveis ao longo do tempo, independentemente da condução.

Houve tendência de decréscimo ao longo do tempo, para a porcentagem de gemas em início de brotação, em todos os tratamentos testados (Figura 1). Entretanto, na testemunha, essa tendência foi linear e, aos 84 dias, ainda foram observadas $27,7 \%$ de gemas em início de brotação, enquanto, nos tratamentos com 
óleo mineral, os valores foram de apenas $10,8 \%, 8,5 \%$ e 5,7\% para as doses de $4 \%, 6 \%$ e $8 \%$, respectivamente. Isso demonstra o efeito positivo do óleo mineral em acelerar a brotação das gemas, enquanto, na testemunha, a proporção entre gemas ainda dormentes $(23,6 \%)$, em início de brotação $(27,7 \%)$ e brotadas $(48,7 \%)$, era semelhante. Em condições de inverno ameno, essa constatação é típica e decorrente da falta de frio para realizar uma eficiente quebra de dormência das gemas laterais (Petri et al., 1996; Petri et al., 2002; Leite, 2005).

Em relação à porcentagem de gemas laterais brotadas, verificou-se tendência de aumento ao longo do tempo em todos os tratamentos (Figura 2). Entretanto, na testemunha, esse aumento foi linear e muito inferior aos tratamentos com óleo mineral. O somatório de horas de frio foi de apenas 141 horas abaixo de $7,2^{\circ} \mathrm{C}$ para o inverno de 2005 , segundo os dados climáticos fornecidos pelo SIMEPAR, o que justifica a desuniformidade e a pouca brotação para esse ano. Aos 49 dias após a aplicação, já foi observada uma superioridade dos tratamentos com óleo mineral com relação à testemunha, onde as concentrações de $6 \%$ e $8 \%$ mostraram respostas idênticas e melhores do que a $4 \%$, em ambos os sistemas de condução. Essa tendência permaneceu constante até a última avaliação aos 84 dias após a aplicação, onde a porcentagem de gemas brotadas foi de $76,1 \%, 81,0 \%$ e $81,8 \%$ para as concentrações de $4 \%, 6 \%$ e $8 \%$, respectivamente. O uso de óleo mineral a $4 \%$ promoveu $41,3 \%$ de gemas vegetativas laterais brotadas na pereira 'Packham's Triumph', enquanto, na testemunha, ocorreu apenas $8,34 \%$ de brotação no ano de 1991, quando o somatório de horas de frio foi de 401 horas em Eldorado do Sul-RS (Francisconi et al., 1992). Já, na safra anterior, com a mesma cultivar no mesmo local, ocorreram 647 horas de frio durante o inverno de 1990, promovendo $55,14 \%$ de brotação na testemunha e $56,8 \%$ com o tratamento com óleo mineral a 4\% (Marodin et al., 1992). Nos anos mais frios, a concentração dos produtos para quebra de dormência pode ser menor, conforme observado em experimento com a macieira 'Gala', onde, em anos com menos de 800 unidades de frio, os melhores resultados para a quebra de dormência foram obtidos com 50 L.ha' ${ }^{-1}$ de óleo mineral mais $5 \mathrm{~L} \mathrm{ha}^{-1}$ de cianamida hidrogenada, enquanto, em anos com mais de 800 unidades de frio, pode-se utilizar $30 \mathrm{~L}^{\mathrm{h} h \mathrm{ha}^{-1}}$ de óleo mineral mais $3 \mathrm{~L}^{\mathrm{h}} \mathrm{ha}^{-1} \mathrm{de}$ cianamida hidrogenada, em volumes de calda que variam de 450 a 1.350 L.ha ${ }^{-1}$. Na quebra de dormência das macieiras 'Gala' e 'Fuji', também foi encontrada maior brotação com óleo mineral a $4 \%$ sozinho, em comparação com a testemunha, mas esse tratamento foi inferior ao uso de óleo mineral associado a outros produtos, como nitrato de cálcio, Erger ${ }^{\circledR}$, calda sulfocálcica e cianamida hidrogenada (Petri, 2005). Já, para a quebra de dormência do pessegueiro 'Flamecrest', uma cultivar de alta exigência em frio, o uso de óleo mineral isolado não apresentou efeito na brotação de gemas vegetativas e floração (Marodin et al., 2002). Para outra cultivar de pessegueiro de alta exigência em frio, a 'Chiripá', a aplicação isolada de óleo mineral a $1 \%$ e $2 \%$ também não apresentou efeito na brotação das gemas vegetativas (Nunes et al., 2001).

Sabe-se que existem diferenças entre gemas vegetativas e floríferas quanto à necessidade de frio para superar a endodormência, sendo que as gemas vegetativas apresentam maior necessidade que as gemas floríferas (Petri et al., 1996; Citadin et al., 2006). Assim, no presente trabalho com pereira 'Hosui', o efeito do óleo mineral, apesar de ter sido eficiente para gemas vegetativas, não foi para as gemas floríferas, diferindo dos resultados obtidos por Citadin et al. (2006) e Marodin et al. (2002). O uso de óleo mineral não aumentou o número de cachos florais. Esse comportamento pode ser entendido, pois, em geral, o número de cachos florais aumenta no ano seguinte à aplicação dos tratamentos de quebra de dormência (Francisconi et al., 1992). O aumento verificado na brotação, possivelmente, propiciará maior formação de ramos frutíferos nos anos subseqüentes.

$\mathrm{O}$ efeito desses produtos é muito específico para cada cultivar, em cada região distinta climaticamente, como ocorreu num experimento com diferentes concentrações de cianamida hidrogenada $(0,25$ a $1 \%)$ mais óleo mineral (3\%), para a quebra de dormência da macieira cv. Eva, em Cambé-PR. Apesar de ser uma cultivar de baixa exigência em frio, a região norte do Estado possui um somatório muito baixo de frio, o que ocasionou uma brotação de apenas $48,8 \%$ das gemas laterais com $1 \%$ de cianamida hidrogenada, aos 50 dias após a aplicação (Roberto et al., 2006). Já, um experimento semelhante com a mesma cultivar, em Ponta Grossa-PR, onde o número de horas de frio foi de $254 \mathrm{em} 2000$, a aplicação de $1 \%$ de cianamida hidrogenada mais $3 \%$ de óleo mineral permitiu a brotação de $70 \%$ das gemas laterais e, com o uso de $1,5 \%$ de cianamida hidrogenada, obtiveram-se $93,57 \%$ de brotação (Cruz Júnior \& Ayub, 2002).

Aos 84 dias após a aplicação do óleo mineral, não foi verificada diferença na brotação das gemas, entre os tipos de condução, para todos os tratamentos (Tabela 1), mas observouse que o efeito do uso do óleo mineral promoveu maior brotação em relação à testemunha e não houve diferença entre as concentrações testadas.

TABELA 1 - Efeito das concentrações de óleo mineral sobre a porcentagem de gemas brotadas de pereira 'Hosui', aos 84 dias após a aplicação, em dois tipos de condução. Pinhais-PR. 2005.

\begin{tabular}{ccc}
\hline & \multicolumn{3}{c}{ Sistema de condução } \\
\hline Óleo mineral & Taça & Líder central modificado \\
\hline Testemunha & $50,07 \mathrm{Ab}$ & $47,42 \mathrm{Ab}$ \\
$4 \%$ & $72,02 \mathrm{Aa}$ & $80,17 \mathrm{Aa}$ \\
$6 \%$ & $73,70 \mathrm{Aa}$ & $88,32 \mathrm{Aa}$ \\
$8 \%$ & $78,02 \mathrm{Aa}$ & $85,55 \mathrm{Aa}$ \\
\hline $\mathrm{CV}(\%)$ & & 16,48 \\
\hline
\end{tabular}

Médias seguidas da mesma letra maiúscula na linha e minúscula na coluna não diferem entre si ,pelo teste de Duncan ao nível de $5 \%$ de probabilidade. 


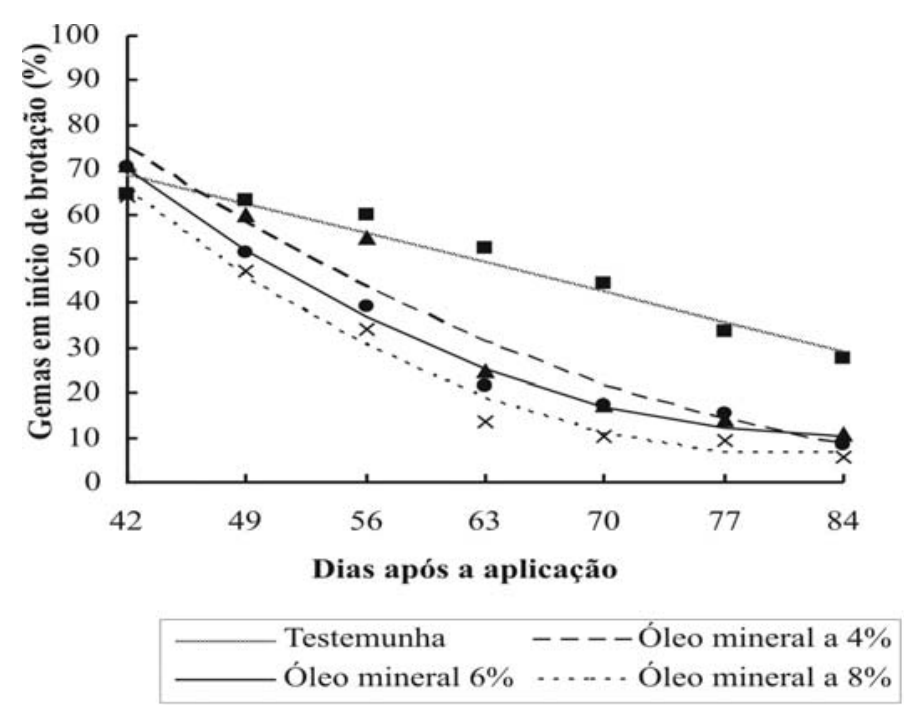

FIGURA 1 - Efeito de concentrações de óleo mineral sobre a porcentagem de gemas em início de brotação, durante um período de 42 a 84 dias após a aplicação, em pereira 'Hosui'. Médias obtidas de plantas conduzidas em líder central modificado e taça. Pinhais-PR. 2005. Testemunha: $Y=108,72-0,94 x$; $\mathrm{R}^{2}=0,95$; Óleo mineral a $4 \%: \mathrm{Y}=223,87-4,53 \mathrm{x}+$ $0,023 \mathrm{x}^{2} ; \mathrm{R}^{2}=0,94$; Óleo mineral a $6 \%$ : $\mathrm{Y}=248,84-$ $5,66 x+0,033 x^{2} ; R^{2}=0,98$; Óleo mineral a $8 \%: Y=$ $262,79-6,35 \mathrm{x}+0,039 \mathrm{x}^{2} ; \mathrm{R}^{2}=0,98$.

\section{CONCLUSÕES}

1-A aplicação de óleo mineral nas concentrações de 4\%, $6 \%$ e $8 \%$ antecipam a brotação de gemas e aumentam a porcentagem de gemas brotadas, sendo as concentrações de $6 \%$ e $8 \%$ mais efetivas.

2-A porcentagem de gemas brotadas após a aplicação do óleo mineral não difere entre os sistemas de condução, em líder central modificado e em taça.

\section{AGRADECIMENTOS}

Os autores agradecem à Secretaria de Estado de Ciência e Tecnologia (SETI) / Governo do Estado do Paraná, pelo apoio financeiro e concessão de bolsa para as alunas de graduação, e ao SIMEPAR, pelos dados climáticos.

\section{REFERÊNCIAS}

ARAUJO, M.M.; FORTES, G.R. de L.; SANTOS FILHO, B.G. Thidiazuron: uma alternativa para superar a dormência de gemas de macieira (Malus domestica Borkh). Revista Brasileira de Fruticultura, Cruz das Almas, v. 13, n. 3, p. 249-253, 1991.

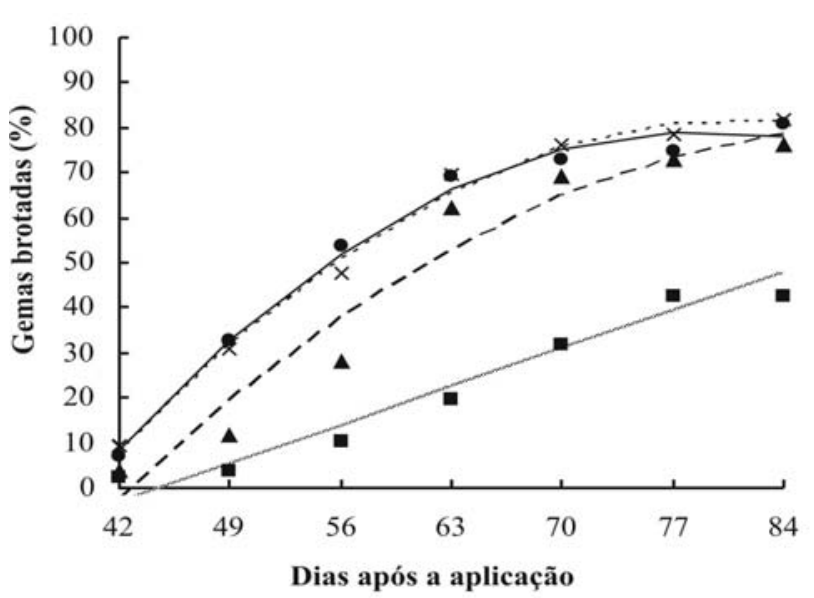

Testemunha _ - - - Óleo mineral a $4 \%$ Óleo mineral $6 \%$.... Óleo mineral a $8 \%$

FIGURA 2 - Efeito de concentrações de óleo mineral sobre a porcentagem de gemas brotadas, durante um período de 42 a 84 dias após a aplicação, em pereira 'Hosui'. Médias obtidas de plantas conduzidas em líder central modificado e taça. Pinhais-PR. 2005. Testemunha: $\mathrm{Y}=-53,75+1,21 \mathrm{x} ; \mathrm{R}^{2}=0,96$; Óleo mineral a $4 \%$ : $Y=-205,7+6,26 x-0,034 x^{2} ; R^{2}$ $=0,94$; Óleo mineral a $6 \%: \mathrm{Y}=-247,01+8,28 \mathrm{x}-$ $0,052 \mathrm{x}^{2} ; \mathrm{R}^{2}=0,98$; Óleo mineral a $8 \%: Y=-233,74+$ $7,76 x-0,047 x^{2} ; R^{2}=0,99$.

BOTELHO, R.V.; AYUB, R.A.; MÜLLER, M.M.L. Somatória de horas de frio e de unidades de frio em diferentes regiões do Estado do Paraná. Scientia Agraria, Curitiba, v. 7, n. 1-2, p. 8996, 2006a.

BOTELHO, R.V.; MÜLLER, M.M.L.; AYUB, R.A. Avaliação do extrato de alho na quebra de dormência de gemas de macieiras 'Royal Gala'. In: ENCONTRO NACIONAL SOBRE FRUTICULTURA DE CLIMA TEMPERADO, 9., 2006. Fraiburgo. Anais... Caçador: Epagri, v. 2 (Resumos), 2006b. p. 7.

CITADIN, I.; BASSANI, M.H.; DANNER, M.A.; MAZARO, S.M.; GOUVÊA, A. Uso de cianamida hidrogenada e óleo mineral na floração, brotação e produção do pessegueiro 'Chiripá'. Revista Brasileira de Fruticultura, Jaboticabal, v. 28, n. 1, p. 3235, 2006.

CRUZ JÚNIOR, A.O.; AYUB, R.A. Quebra de dormência de gemas de macieira cv. Eva tratadas com cianamida hidrogenada. Revista Brasileira de Fruticultura, Jaboticabal, v. 24, n. 2, p. 576-578, 2002.

FAORO, I.D. Cultivares e porta-enxertos. In: EPAGRI. Nashi, a pêra japonesa. Florianópolis: EPAGRI/JICA, 2001. p. 95-138.

FRANCISCONI, A.H.D.; BARRADAS, C.I.N.; MARODIN, G.A.B.; SEIBERT, E. Efeito de óleo mineral, cianamida 
hidrogenada e thidiazuron na quebra de dormência e produção da pereira (Pyrus communis L.) cv. Packham's Triumph. Revista Brasileira de Fruticultura, Cruz das Almas, v.14, n.1, p.161-166, 1992.

LEITE, G.B. Evolução da Dormência e a Heterogeneidade na Brotação. In: ENCONTRO NACIONAL SOBRE FRUTICULTURA DE CLIMA TEMPERADO, 8., 2005. Fraiburgo. Anais... Caçador: Epagri, v. 1 (Palestras), 2005. p. 269-275.

MARODIN, G.A.B.; FRANCISCONI, A.H.D.; GALLOIS, E.S.P. Efeito de produtos químicos na quebra de dormência e produção da pereira (Pyrus communis L.) cv. Packham's Triumph. Revista Brasileira de Fruticultura, Cruz das Almas, v.14, n.1, p.155-160, 1992.

MARODIN, G.A.B.; SARTORI, I.A.; GUERRA, D.S. Efeito da aplicação de cianamida hidrogenada e óleo mineral na quebra de dormência e produção do pessegueiro 'Flamecrest'. Revista Brasileira de Fruticultura, Jaboticabal, v.24, n.2, p.426-230, 2002.

NACHTIGALL, G.R.; CAMELATTO, D. Efeito da mistura de nitrato de cálcio com thidiazuron, cinamida hidrogenada e óleo mineral na superação da dormência de macieiras (Malus domestica, Borkh.) cv. Gala. Revista Brasileira de Fruticultura, Cruz das Almas, v. 19, n. 3, p. 275-282, 1997.

NUNES, J.L.S.; MARODIN, G.A.B.; SARTORI, I.A. Cianamida hidrogenada, thidiazuron e óleo mineral na quebra da dormência e na produção do pessegueiro cv. Chiripá. Revista Brasileira de Fruticultura, Jaboticabal, v. 23, n. 3, p. 493-496, 2001.
PETRI, J.L. Alternativas Para Quebra de Dormência em Fruteiras de Clima Temperado. In: ENCONTRO NACIONAL SOBRE FRUTICULTURA DE CLIMA TEMPERADO, 8., 2005. Fraiburgo. Anais... Caçador: Epagri, v. 1 (Palestras), 2005. p. 303-311.

PETRI, J. L. ; LEITE, G. B. . Consequences of insufficient Winter Chithing on Apple Tree Bud Beak. Acta Horticulturae, p. 53-60, 2004.

PETRI, J.L.; PALLADINI, L.A.; POLA, A.C. Dormência e indução da brotação da macieira. In: EPAGRI. A cultura da macieira. Florianópolis: EPAGRI, 2002. p. 261-298.

PETRI, J.L.; PALLADINI, L.A.; SHUCK, E.; DUCROQUET, J.P.H.J.; MATOS, C.S.; POLA, A.C. Dormência e indução da brotação de fruteiras de clima temperado. Florianópolis: EPAGRI, 1996. 110p. (EPAGRI. Boletim Técnico, 75).

ROBERTO, S. R.; KAGUEYAMA, M. H.; SANTOS, C. E. dos. Indução da brotação da macieira 'Eva' em região de baixa incidencia de frio. Revista Brasileira de Fruticultura, Jaboticabal, v. 28, n. 1, p. 128-130, 2006.

TAIZ, L.; ZEIGER, E. Plant physiology. Sunderland: Sinauer Associates. 1998. 792p. 